PROCEEDINGS OF THE

AMERICAN MATHEMATICAL SOCIETY

Volume 132, Number 1, Pages 305-312

S 0002-9939(03)07018-7

Article electronically published on May 28, 2003

\title{
MONOID OF SELF-EQUIVALENCES AND FREE LOOP SPACES
}

\author{
YVES FÉLIX AND JEAN-CLAUDE THOMAS
}

(Communicated by Paul Goerss)

\begin{abstract}
Let $M$ be a simply-connected closed oriented $N$-dimensional manifold. We prove that for any field of coefficients $l k$ there exists a natural homomorphism of commutative graded algebras $\Gamma: H_{*}\left(\Omega \operatorname{aut}_{1} M\right) \rightarrow \mathbb{H}_{*}\left(M^{S^{1}}\right)$ where $\mathbb{H}_{*}\left(M^{S^{1}}\right)=H_{*+N}\left(M^{S^{1}}\right)$ is the loop algebra defined by Chas and Sullivan. As usual aut $X$ denotes the monoid of self-equivalences homotopic to the identity, and $\Omega X$ the space of based loops. When $k$ is of characteristic zero, $\Gamma$ yields isomorphisms $H_{(1)}^{n+N}\left(M^{S^{1}}\right) \stackrel{\cong}{\rightrightarrows}\left(\pi_{n}\left(\Omega_{\text {aut }} M\right) \otimes l k\right)^{\vee}$ where $\bigoplus_{l=1}^{\infty} H_{(l)}^{n}\left(M^{S^{1}}\right)$ denotes the Hodge decomposition on $H^{*}\left(M^{S^{1}}\right)$.
\end{abstract}

\section{INTRODUCTION}

Let $M$ be a simply connected $N$-dimensional closed oriented manifold with base point $m_{0}$. We denote by $M^{S^{1}}$ the space of free loops on $M$, by $\Omega M$ the space of based loops of $M$ at $m_{0}$, by aut $M$ the monoid of (unbased) self equivalences of $M$, by aut ${ }_{1} M$ the connected component of $I d_{M}$ in aut $M$, and by $H_{*}(-)$ the singular homology functor with coefficients in the fixed field $l k$. The composition of loops induce a commutative graded algebra structure on $H_{*}\left(\Omega_{\text {aut }} M\right)$.

It is convenient to write

$$
\mathbb{H}_{*}(M)=H_{*+N}(M)\left(\operatorname{resp} . \mathbb{H}_{*}\left(M^{S^{1}}\right)=H_{*+N}\left(M^{S^{1}}\right)\right) .
$$

Indeed $\mathbb{H}_{*}(M)$ becomes a commutative graded algebra with the intersection product, and $\mathbb{H}_{*}\left(M^{S^{1}}\right)$ a commuative graded algebra with the loop product defined by Chas and Sullivan [1. The definition of the loop product works as follows: Let $\alpha: \triangle^{n} \rightarrow M^{S^{1}}$ and $\beta: \triangle^{m} \rightarrow M^{S^{1}}$ be simplices of $M^{S^{1}}$ and assume that $q \circ \alpha: \triangle^{n} \rightarrow M$ and $q \circ \beta: \triangle^{m} \rightarrow M$ are transverse in some sense. Then the intersection product $(q \circ \alpha) \cdot(q \circ \beta)$ makes sense, and at each point $(s, t) \in \triangle^{n} \times \triangle^{m}$ such that $q \sigma(s)=q \tau(t)$, the composition of the loops $\alpha(s)$ and $\beta(t)$ can be performed. This gives a chain $\alpha \cdot \beta \in \mathcal{C}_{m+n-N}\left(M^{S^{1}}\right)$ and leads to a commutative and associative multiplication ([1], Theorem 3.3):

$$
\mathbb{H}_{k}\left(M^{S^{1}}\right) \otimes \mathbb{H}_{l}\left(M^{S^{1}}\right) \rightarrow \mathbb{H}_{k+l}\left(M^{S^{1}}\right), \quad a \otimes b \mapsto a \cdot b .
$$

Our first result reads:

Received by the editors May 5, 2002 and, in revised form, August 30, 2002.

2000 Mathematics Subject Classification. Primary 55P35, 55P62, 55P10.

(C)2003 American Mathematical Society 
Theorem 1. The natural map

$$
g: M \times \Omega \text { aut }_{1} M \rightarrow M^{S^{1}}, \quad g(x, \gamma)(t)=\gamma(t)(x),
$$

induces a morphism of commutative graded algebras

$$
H_{*}(g): \mathbb{H}_{*}(M) \otimes H_{*}\left(\operatorname{\Omega aut}_{1} M\right) \rightarrow \mathbb{H}_{*}\left(M^{S^{1}}\right) .
$$

Denote by $\omega$ the fundamental class of $M$ in homology. Then $\omega \in \mathbb{H}_{0}(M)=$ $H_{N}(M) \cong l k \omega$ is the unit of the algebra $\mathbb{H}_{*}(M)$. The homomorphism $H_{*}(g)$ restricts to a morphism of commutative graded algebras

$$
\Gamma: H_{*}\left(\Omega_{\text {aut }_{1}} M\right) \rightarrow \mathbb{H}_{*}\left(M^{S^{1}}\right), \quad \Gamma(a)=H(g)(\omega \otimes a) .
$$

The composition of $\Gamma$ with the Hurewicz map $h: \pi_{*}\left(\operatorname{\Omega aut}_{1} M\right) \otimes l k \rightarrow H_{*}\left(\operatorname{\Omega aut}_{1} M\right)$ is a morphism of graded vector spaces

$$
\Gamma_{1}=\Gamma \circ h: \pi_{*}\left(\operatorname{Raut}_{1} M\right) \otimes l k \rightarrow H_{*+N}\left(M^{S^{1}}\right),
$$

which in turn induces the dual morphism

$$
\Gamma_{1}^{\vee}: H^{*+N}\left(M^{S^{1}}\right) \rightarrow\left(\pi_{*}\left(\operatorname{Raut}_{1} M \otimes l k\right)\right)^{\vee} .
$$

Now recall that $H^{*}\left(M^{S^{1}}\right)$ is isomorphic as a graded vector space with the Hochschild homology of the cochain algebra $\mathcal{C}^{*}(M)([10])$ :

$$
H^{*}\left(M^{S^{1}}\right) \cong H H_{*}\left(\mathcal{C}^{*}(M) ; \mathcal{C}^{*}(M)\right) .
$$

Also recall that if $l k$ is a field of characteristic zero and $A$ is a commutative graded $l k$-algebra, then the Hochschild homology of $A, H H_{*}(A ; A)$, admits a Hodge decomposition ([8]):

$$
\mathbb{H}_{*}(A ; A)=\bigoplus_{l \geq 0}^{\infty} \mathbb{H}_{*}^{(l)}(A ; A)
$$

Since $\mathcal{C}^{*}(M)$ is quasi-isomorphic to a commutative graded differential algebra $A$, we derive from the previous considerations a Hodge decomposition on the free loop space cohomology of $M$,

$$
\mathbb{H}^{*}\left(M^{S^{1}}\right)=\bigoplus_{l \geq 0} \mathbb{H}_{(l)}^{*}\left(M^{S^{1}}\right)
$$

We prove:

Theorem 2. If $k$ is a field of characteristic zero, then

- $\Gamma_{1}: \pi_{*}\left(\Omega\right.$ aut $\left._{1} M\right) \otimes \mathbb{k} \rightarrow \mathbb{H}_{*}\left(M^{S^{1}}\right)$ is injective,

- $\Gamma_{1}^{\vee}: \mathbb{H}_{(1)}^{n}\left(M^{S^{1}}\right) \stackrel{\cong}{\rightrightarrows}\left(\pi_{n}\left(\Omega \text { aut }_{1} M\right) \otimes l k\right)^{\vee}$ is an isomorphism for $n \geq 0$,

- $\Gamma_{1}^{\vee}$ vanishes on the components $\mathbb{H}_{(p)}^{*}\left(M^{S^{1}}\right)$ for $p \geq 2$.

Theorems 1 and 2 are proved respectively in sections 2 and 3 . Section 4 contains examples and final remarks. 


\section{Proof of Theorem 1}

We denote by $q: M^{S^{1}} \rightarrow M$ the free loop space fibration and by $\operatorname{Sect}(q)$ the space of sections of $q$. The composition of loops makes Sect $(q)$ into a monoid with multiplication $\mu$ defined by

$$
\mu(\sigma, \tau)(m)(t)=\left\{\begin{array}{ll}
\sigma(m)(2 t), & t \leq \frac{1}{2}, \\
\tau(m)(2 t-1), & t \geq \frac{1}{2},
\end{array} \quad \sigma, \tau \in \operatorname{Sect}(q), \quad t \in[0,1], m \in M .\right.
$$

Clearly the map $\psi: \Omega\left(\operatorname{aut}_{1} M, i d_{M}\right) \rightarrow \operatorname{Sect}(q)$ defined by

$$
\psi(f)(m)(t)=f(t)(m)
$$

is a homeomorphism of monoids making commutative the diagram

$$
\begin{array}{ccc}
M \times \operatorname{Sect}(q) & \stackrel{e v}{\rightarrow} & M^{S^{1}} \\
1 \times \psi \downarrow & & \| \\
M \times \operatorname{aut}_{1} M & \stackrel{g}{\rightarrow} & M^{S^{1}},
\end{array}
$$

where $e v$ denotes the evaluation map.

To prove Theorem 1, it therefore suffices to establish that the evaluation map $H_{*}(e v): \mathbb{H}_{*}(M) \otimes H_{*}(\operatorname{Sect}(q)) \rightarrow \mathbb{H}_{*}\left(M^{S^{1}}\right)$ is a morphism of graded algebras.

We first remark that Chas and Sullivan prove that the morphism $H_{*}\left(\sigma_{0}\right)$ : $\mathbb{H}(M) \rightarrow \mathbb{H}\left(M^{S^{1}}\right)$, induced by the trivial section $\sigma_{0}$, is a morphism of graded algebras ([1], Proposition 3.4). Therefore the restriction of $H_{*}(e v)$ to $\mathbb{H}_{*}(M)$ is a morphism of graded algebras.

Recall now that the unit of $\mathbb{H}_{*}(M)$ is the fundamental class $\omega \in \mathbb{H}_{0} M=H_{N} M$. Therefore for a cycle $\sum_{i} n_{i} \alpha_{i}$, with $\alpha_{i}: \Delta^{r} \rightarrow \operatorname{Sect}(q), H_{*}(e v)(\omega \otimes \alpha)$ is the homology class of the sum $\sum_{i} n_{i} \alpha_{i}^{\prime}$ where $\alpha_{i}^{\prime}$ denotes the composition

$$
\alpha_{i}^{\prime}: M \times \Delta^{r} \stackrel{i d \times f}{\longrightarrow} M \times \operatorname{Sect}(q) \stackrel{e v}{\longrightarrow} M^{S^{1}} .
$$

Thus let $\alpha: \triangle^{r} \rightarrow \operatorname{Sect}(q)$ and $\beta: \triangle^{s} \rightarrow$ Sect $(q)$ be simplices. Since the simplices $q \circ \alpha^{\prime}$ and $q \circ \beta^{\prime}$ are transverse in $M$, the Chas-Sullivan product

$\alpha^{\prime} \cdot \beta^{\prime}: M \times \Delta^{r} \times \Delta^{s} \stackrel{i d \times \alpha \times \beta}{\longrightarrow} M \times \operatorname{Sect}(q) \times \operatorname{Sect}(q) \stackrel{(e v, e v)}{\longrightarrow} M^{S^{1}} \times{ }_{M} M^{S^{1}} \stackrel{c}{\longrightarrow} M^{S^{1}}$

is well defined, $c$ denoting pointwise composition of loops.

As the multiplication $\mu$ makes commutative the diagram

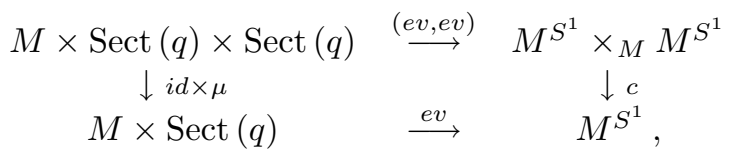

the map $\alpha^{\prime} \cdot \beta^{\prime}$ is equal to $\mu(\alpha, \beta)^{\prime}$. Therefore the restriction of $H_{*}(e v)$ to the component $l k \omega \otimes H_{*}(\operatorname{Sect}(q))$ is also a morphism of algebras.

Finally let $\alpha: \Delta^{r} \rightarrow M$ and $\beta: \Delta^{s} \rightarrow$ Sect $(q)$. Then the simplices $\alpha$ and $q \beta^{\prime}$ are transverse and the Chas-Sullivan product $\alpha \cdot \beta$ is equal to $e v(\alpha \times \beta)$. Therefore $H_{*}(e v)(\alpha) \cdot H_{*}(e v)(\beta)=H_{*}(e v)(\alpha \otimes \beta)$. 


\section{Proof of Theorem 2}

Since $\mathbb{Q} \subset k$ we may as well suppose that $l k=\mathbb{Q}$. Hereafter we will make extensive use of the theory of minimal models in the sense of Sullivan ([12]), for which we refer systematically to [5], $\S 12$. We denote by $(\wedge V, d)$ the minimal model of $M$. By 13] a relative minimal model for the fibration $q: M^{S^{1}} \rightarrow M$ is given by the extension

$$
(\wedge V, d) \hookrightarrow(\wedge V \otimes \wedge s V, D),|s v|=|v|-1, D(v)=d(v), D(s v)=-s(d v),
$$

where $s: \wedge V \rightarrow \wedge V \otimes \wedge s V$ is the unique derivation defined by $s(v)=s v$. The cochain complex $(\wedge V \otimes \wedge s V, D)$ decomposes into a direct sum of complexes

$$
(\wedge V \otimes \wedge s V, D)=\bigoplus_{k \geq 0}\left(\wedge V \otimes \wedge^{k} s V, D\right) .
$$

This induces a new graduation on $H^{*}\left(M^{S^{1}}\right), H^{*}\left(M^{S^{1}}\right)=\bigoplus_{k} H_{(k)}^{*}\left(M^{S^{1}}\right)$ with

$$
H_{(k)}^{*}\left(M^{S^{1}}\right)=H^{*}\left(\wedge V \otimes \wedge^{k} s V, D\right) .
$$

In [14, Vigué proves that this decomposition coincides with the Hodge decomposition of the Hochschild homology $\mathbb{H}_{*}((\wedge V, d) ;(\wedge V, d))$ :

$$
H^{*}\left(\wedge V \otimes \wedge^{k} s V, D\right) \cong \mathbb{H}_{*}^{(k)}((\wedge V, d) ;(\wedge V, d)) .
$$

By the Milnor-Moore Theorem $([1]), H_{*}(\operatorname{Sect}(q) ; \mathbb{Q})$ is isomorphic as a Hopf algebra to the universal enveloping algebra on the graded homotopy Lie algebra $\pi_{*}\left(\Omega\right.$ aut $\left._{1} M\right) \otimes \mathbb{Q}$. Thus Theorem 2 in the Introduction is a direct consequence of Theorem 3 below.

Theorem 3. Let

$$
\Phi_{1}: \pi_{*}(\operatorname{Sect}(q)) \otimes \mathbb{Q} \rightarrow \mathbb{H}_{*}\left(M^{S^{1}} ; \mathbb{Q}\right)
$$

denote the restriction of $H_{*}(e v)$ to $\omega \otimes \pi_{*}(\operatorname{Sect}(q)) \otimes \mathbb{Q}$. Then,

- $\Phi_{1}$ is an injective morphism,

- the dual map $\Phi_{1} \vee$ vanishes on each $H_{(p)}^{*}\left(M^{S^{1}} ; \mathbb{Q}\right), p \geq 2$, and induces an isomorphism $\bigoplus_{q>N} H_{(1)}^{q}\left(M^{S^{1}} ; \mathbb{Q}\right) \cong \pi_{*}(\operatorname{Sect}(q))^{\vee}$.

Proof. We first construct a quasi-isomorphism $\rho:(\wedge V, d) \rightarrow(A, d)$ with $(A, d)$, a commutative differential graded algebra satisfying $A^{0}=\mathbb{Q}, A^{1}=0, A^{>N}=0$, $A^{N}=\mathbb{Q} \omega$, and $\operatorname{dim} A^{i}<\infty$ for all $i$.

For this we denote

$$
Z^{k}=\operatorname{Ker}\left(d:(\wedge V)^{k} \rightarrow(\wedge V)^{k+1}\right),
$$

and we choose a supplement $S^{k}$ of $Z^{k}$ in $(\wedge V)^{k}$ :

$$
(\wedge V)^{k}=Z^{k} \oplus S^{k}
$$

The quotient $(\wedge V)^{N} /\left(S^{N} \oplus d S^{N-1}\right) \cong H^{N}(M)$ has dimension one. Since $V^{1}=0$, the subcomplex $I=S^{N-1} \oplus d S^{N-1} \oplus S^{N} \oplus(\wedge V)^{>N}$ is an acyclic ideal in $(\wedge V, d)$. Therefore the natural projection $\rho:(\wedge V, d) \rightarrow(\wedge V / I, d)$ is a quasi-isomorphism of differential graded algebras. We define $(A, d)=(\wedge V / I, d)$.

The homomorphism $\rho$ extends to a quasi-isomorphism $\rho \otimes 1:(\wedge V \otimes \wedge s V, D) \rightarrow$ $(A \otimes \wedge s V, D)$ with $D(a \otimes s v)=d(a) \otimes s v-(-1)^{|a|} a \cdot(\rho \otimes 1)(D s v)$. The complex $(A \otimes \wedge s V, D)$ also decomposes into the direct sum of the complexes $\left(A \otimes \wedge^{k} s V, D\right)$. 
Denote by $\left(a_{i}\right), i=1, \ldots, n$, a homogeneous linear basis of $A$ with $a_{n}=\omega$, and by $\left(a_{i}^{\vee}\right)$ the dual basis, i.e. the linear basis of $A^{\vee}=\operatorname{Hom}(A, \mathbb{Q})$ such that

$$
\left\langle a_{i}^{\vee}, a_{j}\right\rangle=\delta_{i j} .
$$

In 9], Haefliger proved that a model for the evaluation map ev $: M \times \operatorname{Sect}(q) \rightarrow$ $M^{S^{1}}$ is given by the morphism

$$
\theta:(A \otimes \wedge s V, D) \rightarrow(A, d) \otimes\left(\wedge\left(A^{\vee} \otimes s V\right), \delta\right), \quad \theta(a \otimes s v)=\sum_{i} a a_{i} \otimes\left(a_{i}^{\vee} \otimes s v\right) .
$$

Since $D(s V) \subset A \otimes s V$ and $\theta$ is a morphism of differential graded algebras, then $\delta\left(A^{\vee} \otimes s V\right) \subset A^{\vee} \otimes s V$. We now fix some notations:

- $\rho_{1}:\left(\wedge\left(A^{\vee} \otimes s V\right), \delta\right) \rightarrow\left(A^{\vee} \otimes s V, \delta\right)$ denotes the projection on the complex of indecomposable elements,

- $P:(A, d) \rightarrow(\mathbb{Q} \omega, 0)$ is the homogeneous projection onto the component of degree $N$,

- $\pi_{1}:(A \otimes \wedge s V, D) \rightarrow(A \otimes s V, D)$ is the canonical projection on the subcomplex $(A \otimes s V, D)$.

The dual of $\Phi_{1}$,

$$
\Phi_{1}^{\vee}: H^{*+d}\left(M^{S^{1}} ; \mathbb{Q}\right) \rightarrow\left(\pi_{*}(\operatorname{Sect}(q)) \otimes \mathbb{Q}\right)^{\vee},
$$

therefore coincides with $H^{*}\left(P \otimes \rho_{1}\right) \circ H^{*}(\theta)$ :

$$
(A \otimes \wedge s V, D) \stackrel{\theta}{\longrightarrow}(A, d) \otimes\left(\wedge\left(A^{\vee} \otimes s V\right), \delta\right) \stackrel{P \otimes \rho_{1}}{\longrightarrow} \mathbb{Q} \omega \otimes\left(A^{\vee} \otimes s V, \delta\right),
$$

and vanishes on $\left(A \otimes \wedge^{\geq 2} s V, D\right)$.

Lemma. The duality map $\Delta: A \rightarrow A^{\vee}$ defined by

$$
\langle\Delta(a), b\rangle=P(a b) \in \mathbb{Q} \omega \cong \mathbb{Q}
$$

extends into a quasi-isomorphism of complexes

$$
\Delta \otimes 1:(A \otimes s V, D) \rightarrow\left(A^{\vee} \otimes s V, \delta\right) .
$$

Proof. Denote by $\alpha_{i j}^{k}$ and $\beta_{i}^{j}$ rational numbers defined by the relations

$$
\left\{\begin{array}{l}
a_{i} \cdot a_{j}=\sum_{k} \alpha_{i j}^{k} a_{k}, \\
d\left(a_{i}\right)=\sum_{j} \beta_{i}^{j} a_{j} .
\end{array}\right.
$$

Recall that $\left\{a_{i}^{\vee}\right\}_{i}$ denotes the dual basis of $\left\{a_{i}\right\}_{i}$. Then straightforward computations show that

- $d\left(a_{i}^{\vee}\right)=-(-1)^{\left|a_{i}\right|} \sum_{j} \beta_{j}^{i} a_{j}^{\vee}$.

- $\sum_{r} \alpha_{i j}^{r} \alpha_{r k}^{t}=\sum_{s} \alpha_{j k}^{s} \alpha_{i s}^{t}$, for $i, j, k, t=1, \ldots, n$ (associativity of the multiplication law).

- $\sum_{r} \alpha_{i j}^{r} \beta_{r}^{s}=\sum_{t} \beta_{i}^{t} \alpha_{t j}^{s}+(-1)^{\left|a_{i}\right|} \sum_{l} \beta_{j}^{l} \alpha_{i l}^{s}$, for $i, j, l=1, \ldots, n$ (compatibility of the differential $d$ with the multiplication).

- $\delta\left(a_{j}^{\vee} \otimes s v\right)=(-1)^{\left|a_{j}\right|}\left[\sum_{i, l} \alpha_{i l}^{j}\left(a_{l}^{\vee} \otimes s v_{i}\right)-\sum_{r} \beta_{r}^{j}\left(a_{r}^{\vee} \otimes s v\right)\right]$.

- $\Delta\left(a_{i}\right)=\sum_{j} \alpha_{i j}^{n} a_{j}^{\vee}$. 
The duality morphism has degree $N$. A standard computation then shows that

$$
\delta \circ(\Delta \otimes 1)=(-1)^{N}(\Delta \otimes 1) \circ d .
$$

Since $H^{*}(M)$ is a Poincaré duality algebra and since $H^{*}(\Delta): H^{*}(M) \rightarrow H_{*}(M)$ is the Poincaré duality, $\Delta \otimes 1$ is a quasi-isomorphism.

End of the proof of Theorem 3. It is easy to check the commutativity of the following diagram of complexes:

$$
\begin{aligned}
& (A \otimes \wedge s V, D) \stackrel{\theta}{\longrightarrow}(A, d) \otimes\left(\wedge\left(A^{\vee} \otimes s V\right), \delta\right) \stackrel{P \otimes \rho_{1}}{\longrightarrow} \mathbb{Q} \omega \otimes\left(A^{\vee} \otimes s V, \delta\right) \\
& \pi_{1} \downarrow \quad \uparrow 1 \otimes(\Delta \otimes 1) \\
& (A \otimes s V, D) \quad \stackrel{\sigma}{\longrightarrow} \quad \mathbb{Q} \omega \otimes(A \otimes s V, D),
\end{aligned}
$$

with $\sigma(a \otimes s v)=\omega \otimes a \otimes s v$. By the above lemma, $H_{*}(1 \otimes \Delta \otimes 1)$ is an isomorphism. Therefore $H^{*}\left((1 \otimes(\Delta \otimes 1)) \circ \sigma \circ \pi_{1}\right)$ is surjective and this implies the surjectivity of $\Phi_{1}^{\vee}=H_{*}\left(P \otimes \rho_{1}\right) \circ H^{*}(\theta)$.

\section{EXAmples AND FURTher COMments}

Remark 1. The morphism $\Gamma: H_{*}\left(\Omega\right.$ aut $\left._{1} M\right) \rightarrow H_{*}\left(M^{S^{1}}\right)$ is not injective in general, as we shall now explain.

Denote by $e v_{0}:$ aut $_{1} M \rightarrow M$ the evaluation at the base point. The image of the morphism $\pi_{n}\left(e v_{0}\right): \pi_{n}\left(\right.$ aut $\left._{1} M\right) \rightarrow \pi_{n} M$ is known as the $n$-th Gottlieb group of $M, G_{n}(M)$ ([5]). Since $\Omega e v_{0}: \Omega$ aut $_{1} M \rightarrow \Omega M$ is an H-map, $H_{*}\left(\Omega e v_{0} ; \mathbb{Q}\right)=$ $U\left(\pi_{*}\left(\Omega e v_{0}\right) \otimes \mathbb{Q}\right)$ is the enveloping algebra on $\pi_{*}\left(\Omega e v_{0}\right) \otimes \mathbb{Q}$, whose image is the enveloping algebra on the abelian graded Lie algebra $\bar{G}_{*}(X)$ that corresponds by duality to $G_{*}(X) \otimes \mathbb{Q}$.

Denote by $I: \mathbb{H}_{*}\left(M^{S^{1}}\right) \rightarrow H_{*}(\Omega M)$ the intersection morphism defined in ([1], Proposition 3.4), and let $\psi$ be defined as in the beginning of section 2. The commutativity of the following diagram

$$
\begin{array}{ccc}
H_{*}\left(\Omega \operatorname{aut}_{1} M\right) & \stackrel{\pi_{*}(\psi)}{\longrightarrow} & H_{*}(\operatorname{Sect}(q)) \\
H_{*}\left(\Omega e v_{0}\right) \downarrow & & \downarrow H_{*}(e v)(\omega \otimes-) \\
H_{*}(\Omega M) & \stackrel{I}{\leftarrow} & \mathbb{H}_{*}\left(M^{S^{1}}\right)
\end{array}
$$

shows that the image of $I \circ \Phi_{1}$ is the universal enveloping algebra on $\bar{G}_{*}(X)$.

On the other hand, the kernel of $I$ is a nilpotent ideal with nilpotency index less than or equal to $N$ ([6]).

Now consider the manifold $M=S^{3} \times S^{3} \times S^{11}$. A simple computation using minimal models shows that $\pi_{5}\left(\right.$ aut $\left._{1} M\right) \otimes \mathbb{Q} \neq 0$ and $G_{5}(M) \otimes \mathbb{Q}=0$. Then denote by $x$ a nonzero element in $\pi_{4}\left(\Omega\right.$ aut $\left._{1} M\right) \otimes \mathbb{Q}$. Since $H_{*}\left(\Omega\right.$ aut $\left._{1} M ; \mathbb{Q}\right)$ is a free commutative graded algebra, some power of $x$ belongs in the kernel of $\Gamma$.

Remark 2. In 2] Cohen and Jones prove that $\mathbb{H}_{*}\left(M^{S^{1}}\right)$ is isomorphic as an algebra to the Hochschild cohomology $H H^{*}\left(\mathcal{C}^{*}(M), \mathcal{C}^{*}(M)\right)$. On the other hand, in [7], Gatsinzi establishes for any space $M$ an algebraic isomorphism between $\pi_{*}\left(\right.$ aut $\left._{1} M\right) \otimes \mathbb{Q}$ and a sub-vector space of $H H^{*}\left(\mathcal{C}^{*}(M), \mathcal{C}^{*}(M)\right)$. Our Theorem 2 relates these two results. 
Problem. We would like to know if the homomorphism

$$
\Gamma: \mathbb{H}_{*}(M) \otimes H_{*}\left(\operatorname{Saut}_{1} M\right) \rightarrow \mathbb{H}_{*}\left(M^{S^{1}}\right)
$$

is surjective. It is true for example when $M=\mathbb{C} P^{2 N}$. When $\Gamma$ is surjective there is a strong connection between the behaviour of the sequences of Betti numbers $\operatorname{dim} H_{i}\left(M^{S^{1}}\right)$ and $\operatorname{dim} \pi_{i}($ aut $M) \otimes \mathbb{Q}$.

Example 1. Let $G$ be a Lie group. The minimal model of $G$ is $(\wedge V, 0)$ with $V$ finite dimensional and concentrated in odd degrees $([5, \S 12(\mathrm{a}))$. Therefore a model of the free loop space $G^{S^{1}}$ is $(\wedge V \otimes \wedge s V, 0)$ and the Haefliger model for the space Sect $(q)$ is $\left(\wedge\left((\wedge V)^{\vee} \otimes s V\right), 0\right)$. Since the model $\theta$ of the evaluation map $e v$ is injective, $H_{*}(e v): H_{*}(M) \otimes H_{*}(\operatorname{Sect}(q)) \rightarrow H_{*}\left(M^{S^{1}}\right)$ is surjective. This implies the existence of an isomorphism of graded algebras,

$$
\mathbb{H}_{*}\left(M^{S^{1}}\right) \cong \mathbb{H}_{*}(M) \otimes H_{*}(\Omega M) .
$$

Here the multiplication on the right is the product of the intersection product on $\mathbb{H}_{*}(M)$ with the usual Pontryagin product on $H_{*}(\Omega M)$.

Example 2. Let us assume that $M$ is a $\mathbb{Q}$-hyperbolic space satisfying either $\left(H^{+}(M)\right)^{3}=0$ or $\left(H^{+}(M)\right)^{4}=0$, and $M$ is a coformal space.

Recall that a space $M$ is $\mathbb{Q}$-hyperbolic if $\operatorname{dim} \pi_{*}(M) \otimes \mathbb{Q}=\infty$ and is coformal if the differential graded algebras $\mathcal{C}_{*}(\Omega M)$ and $\left(H_{*}(\Omega M), 0\right)$ are quasi-isomorphic. Under the above hypothesis, in [15] Vigué proves that there exist an integer $n_{0}$ and some constants $C_{1} \geq C_{2}>1$ such that

$$
C_{2}^{n} \leq \sum_{i=1}^{n} \operatorname{dim} H_{(1)}^{i}\left(X^{S^{1}}\right) \leq C_{1}^{n}, \text { for all } n \geq n_{0} .
$$

We deduce from Theorem 3 that the same relations hold for the sequence of dimensions of $\pi_{i}($ aut $M) \otimes \mathbb{Q}$, i.e., in both cases the sequences of Betti numbers have exponential growth.

\section{REFERENCES}

1. M. Chas and D. Sullivan, String topology, preprint math GT/9911159.

2. R. Cohen and J. Jones, A homotopy theoretic realization of string topology, Math. Ann. 324 (2002) 773-798.

3. R. Cohen, J. D.S. Jones and J. Yan, The loop homology of spheres and projective spaces, preprint, February 2002.

4. Y. Félix and J.-C. Thomas, The monoid of self-homotopy equivalences of some homogeneous spaces, Expositiones Mathematicae 12 (1994) 305-321. MR 95i:55013

5. Y. Félix, S. Halperin and J.-C. Thomas, Rational Homotopy Theory, Graduate Texts in Mathematics 205, Springer-Verlag (2000). MR 2002d:55014

6. Y. Félix, J.-C. Thomas and M. Vigué-Poirrier, Structure of the loop homology of a compact manifold, preprint, March 2002.

7. J.-B. Gatsinzi, The homotopy Lie algebra of classifying spaces, Journal of Pure and Applied Algebra 120 (1997) 281-289. MR 98g:55015

8. M. Gerstenhaber and S. D. Schack, A Hodge type decomposition for commutative algebras, J. Pure Appl. Algebra 48 (1987) no. 3, 229-247. MR 88k:13011

9. A. Haefliger, Rational homotopy of the space of sections of a nilpotent bundle, Trans. Amer. Math. Soc. 273 (1982), 609-620. MR 84a:55010

10. J.D.S. Jones, Cyclic homology and equivariant homology, Invent. Math. 87 (1987), $403-423$. MR 88f:18016

11. J. Milnor and J.C. Moore, On the structure of Hopf algebras, Annals of Math. 81 (1965) 211-264. MR 30:4259 
12. D. Sullivan, Infinitesimal computations in topology, Publ. Math. IHES 47 (1978) 269-331. MR 58:31119

13. D. Sullivan and M. Vigué, The homology theory of the closed geodesic problem, J. Differential Geom. 11 (1976) 633-634. MR 56:13269

14. M. Vigué-Poirrier, Décompositions de l'homologie cyclique des algèbres différentielles graduées commutatives, $K$-Theory 4 (1991) 399-410. MR 92e:19004

15. M. Vigué-Poirrier, Homotopie rationnelle et croissance du nombre de géodésiques fermées, Ann. Scient. Ecole Norm. Sup. 17 (1984) 413-431. MR 86h:58027

Département de Mathématique, Université Catholique de Louvain, 2, Chemin du CyClotron, 1348 Louvain-La-Neuve, Belgium

E-mail address: felix@math.ucl.ac.be

Faculté des Sciences, Université D’Angers, 2, Boulevard Lavoisier, 49045 Angers, FRANCE

E-mail address: thomas@univ-angers.fr 